\title{
Gamma-rays signature of dark matter in the CTA era: status and prospects
}

\author{
Aldo Morselli* \\ INFN Roma Tor Vergata \\ E-mail: aldo.morselli@roma2.infn.it
}

Detection of gamma rays and cosmic rays from the annihilation or decay of dark matter particles is a promising method for identifying dark matter, understanding its intrinsic properties, and mapping its distribution in the universe. I will review recent results from the Fermi Gamma-ray Space Telescope and other space-based experiments, and highlight the constraints these currently place on particle dark matter models. I will also discuss the prospects for indirect searches to robustly identify or exclude a dark matter signal using upcoming experiments at energies below Fermi ( e-ASTROGAM ) and above Fermi: Magic, H.E.S.S. VERITAS and finally CTA.

In the last decades a vaste amount of evidence for the existence of dark matter has been accumulated. At the same time, many efforts have been undertaken to try to identify what dark matter is. Indirect searches look at places in the Universe where dark matter is believed to be abundant and seek for possible annihilation or decay signatures.

At high energies, the Cherenkov Telescope Array (CTA) represents the next generation of imaging Cherenkov telescopes and, with one site in the Southern hemisphere and one in the Northern hemisphere, will be able to observe all the sky with unprecedented sensitivity and angular resolution above a few tens of $\mathrm{GeV}$. The CTA Consortium will undertake an ambitious program of indirect dark matter searches for which we report here the brightest prospects.

At lower energies, the energy range between 1 and $100 \mathrm{MeV}$ is an experimentally very difficult range and remained uncovered since the time of COMPTEL. New instruments can address all astrophysics issues left open by the current generation of instruments. In particular, a good angular resolution in the energy range $10 \mathrm{MeV}-1 \mathrm{GeV}$ is crucial to resolve patchy and complex features of diffuse sources in the Galaxy and in the Galactic Centre as well as increasing the point source sensitivity. This instrument addresses scientific topics of great interest to the community, with particular emphasis on multifrequency correlation studies involving radio, optical, IR, X-ray, soft gamma-ray and $\mathrm{TeV}$ emission. The possibility to study not only the pair production regime but also the Compton regime with this kind of detectors is currently under investigation and it is another possible very interesting breaktrought.

2nd World Summit: Exploring the Dark Side of the Universe

25-29 June, 2018

University of Antilles, Pointe-Ã $\breve{a}$-Pitre, Guadeloupe, France

\footnotetext{
* Speaker.
} 


\section{The high enery frontier}

The existence of dark matter (DM) in our Universe is well established, but its nature is at present still unknown. Multiple hypotheses endure as to the character of dark matter and for the most popular models discussed CTA has a unique chance of discovery. Among the most promising particle candidates are Weakly Interacting Massive Particles (WIMPs), which typically can self-annihilate to produce prompt or secondary gamma-rays during the annihilation. If WIMPs are produced thermally in the early Universe then the current self-annihilation cross-section has a natural value of approximately $3 \cdot 10^{-26} \mathrm{~cm}^{3} \mathrm{~s}^{-1}$. WIMPs models, such as the supersymmetric neutralino, give predictions for gamma-ray energy spectra from the annihilations, which are crucial inputs, together with the DM distribution in the observed target, to estimate prospects for the sensitivity of indirect searches [1].

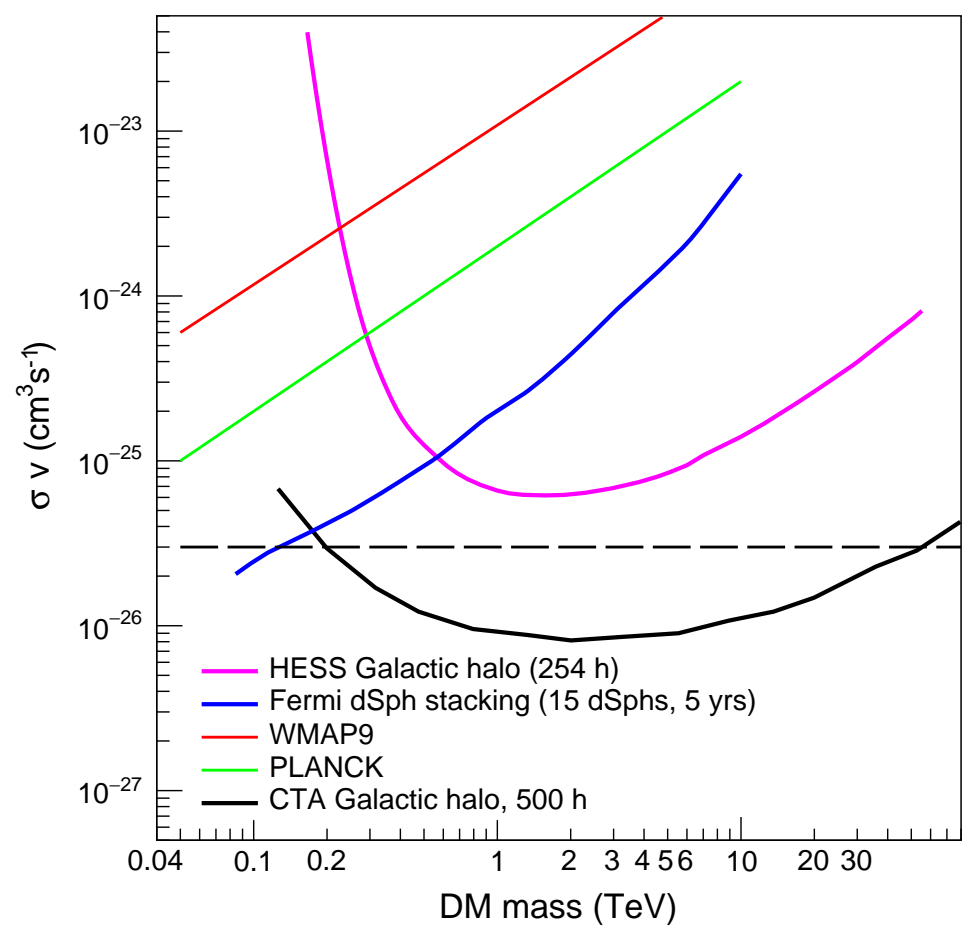

Figure 1: Current best limits on the annihilation cross-section from indirect detection (Fermi-LAT dwarf spheroidal galaxies stacking analysis, $W^{+} W^{-}$channel [5], H.E.S.S. Galactic halo $W^{+} W^{-}$channel [7]) and cosmic microwave background (WMAP and Planck $b \bar{b}$ channel [6]) experiments compared with the projected sensitivity for CTA from observations of the Galactic halo for the Einasto profile, $W^{+} W^{-}$channel. The expectation for CTA is optimistic as it includes only statistical errors. The effect of the Galactic diffuse emission can affect the results by $\sim 50 \%$. The dashed line shows the thermal relic cross section [4].

The priority for the CTA DM program is to discover the nature of DM. The publication of limits following non-detection would certainly happen, but in planning the observational strategy, the priority of discovery drives the programme. The possibility of discovery should be considered 
in the light of model predictions where the minimum goal for searches within the most widely considered models is the thermal cross-section and CTA can probe DM scenarios out of reach for collider searches and direct detection experiments. The principal target for DM observations in CTA is the Galactic halo. This observation will be taken within several degrees of the Galactic centre with the Galactic centre and the most intense diffuse emission regions removed from the analysis. With a cuspy DM profile, observations of 500 hours in this region provide sensitivities below the thermal cross-section and give a significant chance of discovery in some of the most popular models for WIMPs. Since the DM density in the Galactic halo is far from certain, and considering the irreducible background from cosmic rays and the galactic diffuse emission there, other targets are also proposed for observation. Among these secondary targets, the first to be observed will be dwarf spheroidal satellite galaxies with 100 hours per year proposed. Beyond these two observational targets, alternatives will be considered closer to the actual date of CTA operations. New sky surveys such as the Dark Energy Survey (DES) [2] will extend the knowledge of possible sites of large DM concentrations and a detailed study of the latest data will be made to continuously select the best targets for DM searches in CTA. Among these new possible targets are newly discovered candidate dwarf galaxies and not yet discovered DM clumps (pure DM overdensities without baryonic counterpart and thus deprived of any stellar activity) which could be very promising sources if their locations are identified a priori by their gravitational effects. Beyond the targets proposed for observations in the present Key Science Projects (KSP), the data from the Large Magellanic Cloud (LMC) and from the clusters of Galaxies KSPs will also be used to search for DM. Furthermore, the data from the Galactic plane and extragalactic surveys might give hints of gamma-ray sources which do not have counterparts in multi-wavelength data and which could be pursued as DM targets. The DM Programme is very well suited to being carried out in the Core Programme by the CTA Consortium. The observations require a large amount of time with a significant chance of major discovery but with a clear risk of a null detection. For the Galactic halo the observation time used will also be of great use for astrophysics. In the phase between now and the actual operation of CTA, much will evolve in the knowledge of DM distributions in the various targets. Further detailed work is needed to understand the systematics in the backgrounds especially in the Galactic halo that looks the most promising target up to now. As shown in Figure 1, the deep exposure of the Galactic centre region will allow CTA to reach a sensitivity to a thermal relic WIMP over a wide mass region, thus nicely complementing searches done with the Fermi satellite, at the Large Hadron Collider and by direct-detection experiments. Additional dark matter targets include dwarf spheroidal galaxies, the LMC and the Perseus cluster. The effect of systematics is drastically reduced for dwarf spheroidal galaxies compared to the extended Galactic Halo, explaining the significant interest in observations of dwarfs

\section{The low enery frontier}

In Figure 2 is shown the energy range covered by present and future experiments in $\mathrm{X}$ and gamma-ray astrophysics versus the time of operation. There is an energy range below the Fermi and Agile energies that is not covered by any experiments.

In this energy range a new instrument like Gamma-Light [8] or e-ASTROGAM [9, 10] is needed 


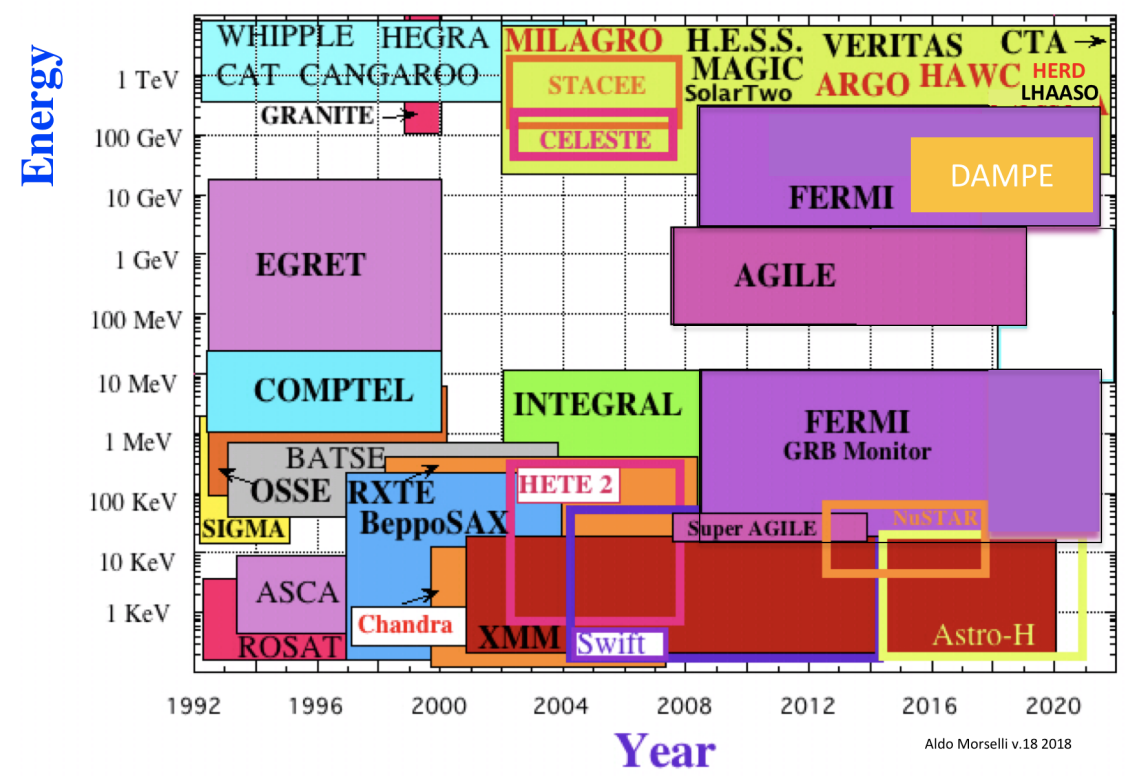

Figure 2: Timeline schedule versus the energy range covered by present and future detectors in $\mathrm{X}$ and gamma-ray astrophysics.

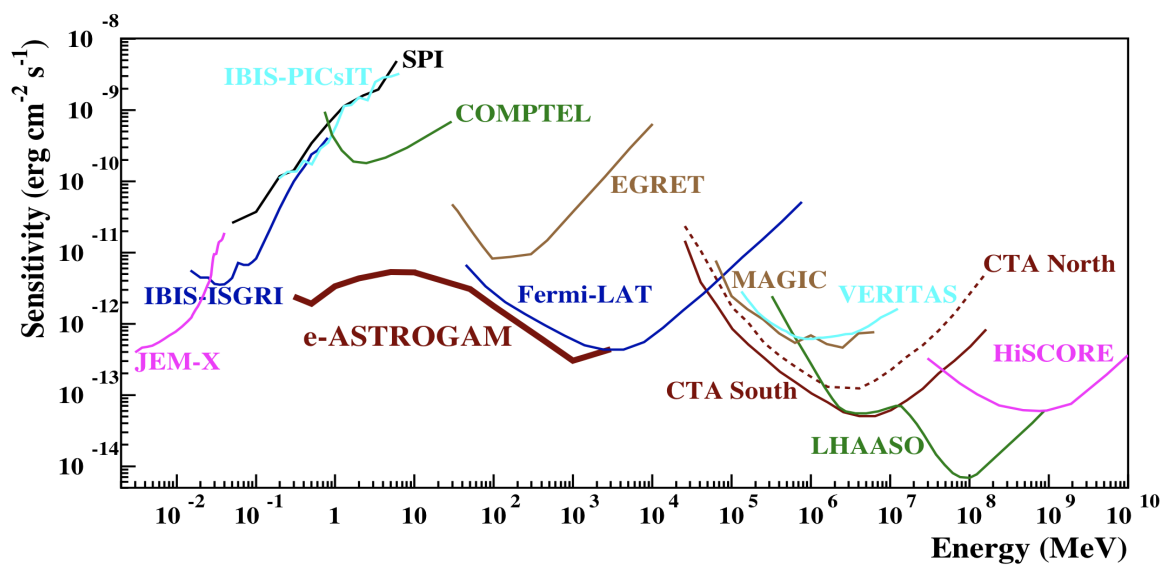

Figure 3: Point source continuum sensitivity of different $X$ and $\gamma$-ray instruments compared with eASTROGAM .

The sensitivity of e-ASTROGAM is shown in figure 3 at $3 \sigma$ for an effective exposure of 1 year of a high Galactic latitude source. The curves for INTEGRAL/JEM-X, IBIS (ISGRI and PICsIT), and SPI are for an effective observation time $T_{\mathrm{obs}}=1 \mathrm{Ms}$. The COMPTEL and EGRET sensitivities are given for the observing time accumulated during the whole duration of the CGRO mission $\left(\mathrm{T}_{o b s}\right.$ $\sim 9$ years). The Fermi-LAT sensitivity is for a high Galactic latitude source and $\mathrm{T}_{\text {obs }}=10$ years . For MAGIC, VERITAS (sensitivity of H.E.S.S. is similar) and CTA the sensitivities are given for 
$\mathrm{T}_{o b s}=50$ hours. For HAWC $T_{\mathrm{obs}}=5 \mathrm{yr}$, for LHAASO $T_{\mathrm{obs}}=1 \mathrm{yr}$, and for HiSCORE $T_{\mathrm{obs}}=1000$ h.

\section{Conclusion}

Detection of gamma rays from the annihilation or decay of dark matter particles is a promising method for identifying dark matter, understanding its intrinsic properties, and mapping its distribution in the universe (in synergy with the experiments at the LHC and in the underground laboratories). In the future it would be extremely important to extend the energy range of experiments at lower energies (compared to the Fermi energies) ( e-ASTROGAM ) and higher energies (HAWC, Dampe, HERD, Gamma-400, CTA, LHAASO)

Beyond the three major "core science" topics, the e-ASTROGAM mission can be also conceived and communicated as a threefold mission, representing: 1) the missing, MeV-energy, piece of the multiwaveband/multimessenger puzzle placed between ATHENA and CTA; 2) the ability to provide data for different science communities (from stellar/nuclear astrophysicists to high-energy astrophysicists, to fundamental/particle physicists); 3) both an established technology with minor risks and a full use of this (double sided detectors, two-process detection) with a full capitalization of silicon detectors in space.

\section{References}

[1] A.Morselli, Constraints on Dark Matter with Gamma-Ray Experiments and Future Observational Strategies, Front. Phys. 5:3. (7pp) 2017, doi: 10.3389/fphy.2017.00003.

[2] Dark Energy Survey Collaboration: T. Abbott et al., The Dark Energy Survey: more than dark energy - an overview, Mon.Not.Roy.Astron.Soc. 460 (2016) no. 2, 1270-1299 [arXiv:1601.00329]

[3] CTA Consortium, Science with the Cherenkov Telescope Array, World Scientific, https://doi.org/10.1142/10986 [arXiv:1709.07997]

[4] A.Morselli, The Dark Matter Programme of the Cherenkov Telescope Array, PoS(ICRC2017)921 [arXiv:1709.01483]

[5] M.Ackermann et al., [Fermi Coll.] Searching for Dark Matter Annihilation from Milky Way Dwarf Spheroidal Galaxies with Six Years of Fermi-LAT Data, PRL 115, 231301 (2015) [arXiv:1503.02641]

[6] Planck Collaboration: P. A. R. Ade et al., Planck 2015 results. XIII. Cosmological parameters A\&A 594, A13 (2016) [arXiv:1502.01589]

[7] H.E.S.S. Collaboration (2017). Search for dark matter annihilations towards the inner Galactic halo from 10 years of observations with H.E.S.S., Phys. Rev. Lett. 117, 111301 [arXiv:1607.08142]

[8] A.Morselli et al., Gamma-Light: High-Energy Astrophysics above $10 \mathrm{MeV}$, Nuclear Physics B 239-240 (2013) 193-198 [arXiv:1406.1071]

[9] A. De Angelis et al., The e-ASTROGAM mission (exploring the extreme Universe with gamma rays in the MeV-GeV range, Experimental Astronomy Vol. 44, Issue 1 (58 pp) [arXiv:1611.02232]

[10] A. De Angelis et al., Science with e-ASTROGAM, Journal of High Energy Astrophysics Volume 19, August 2018, Pages 1-106 https://doi.org/10.1016/j.jheap.2018.07.001 [arXiv:1711.01265] 